\title{
Fabric ation of Poly(methyl methacrylate) Microfluidic Chips by Atmospheric Molding
}

\author{
Alexander Muck, J r., ${ }^{\dagger}$ J oseph Wang, ${ }^{*, \dagger}$ Michael J acobs, ${ }^{\dagger, \ddagger}$ Gang Chen, ${ }^{\dagger}$ Madhu Prakash Chatrathi, ${ }^{\dagger}$ \\ Vlastimil J urka, ${ }^{\S}$ Zdeněk Výborný, ${ }^{\$}$ Scott D. Spillman, ${ }^{\dagger}$ Gautham Sridharan, ${ }^{\dagger}$ and Michael J . Sch ${ }^{\prime}$ 'ning $^{\ddagger}$ \\ Department of Chemistry and Biochemistry, New Mexico State University, Las Cruces, New Mexico 88003, Institute of \\ Thin Films and Interfaces, Research Center Jülich, 524 25, Germany, and Institute of Physics, Academy of Sciences of the \\ Czech Republic, Prague 6, 162 53, Czech Republic
}

A greatly simplified method for fabricating poly(methyl methacrylate) (PMMA) separation microchips is introduced. The new protocol relies on UV-initiated polymerization of the monomer solution in an open mold under ambient pressure. Silicon microstructures are transferred to the polymer substrate by molding a methyl methacrylate solution in a sandwich (silicon master/ Teflon spacer/ glass plate) mold. The chips are subsequently assembled by thermal sealing of the channel and cover plates. The new fabrication method obviates the need for specialized replication equipment and reduces the complexity of prototyping and manufacturing. Variables of the fabrication process were assessed and optimized. The new method compares favorably with common fabrication techniques, yielding high-quality devices with welldefined channel and injection-cross structures, and highly smoothed surfaces. Nearly 100 PMMA chips were replicated using a single silicon master, with high chipto-chip reproducibility (relative standard deviations of 1.5 and $4.7 \%$ for the widths and depths of the replicated channels, respectively). The relatively high EOF value of the new chips $\left(2.12 \times 10^{-4} \mathrm{~cm}^{2} \cdot \mathrm{V}^{-1} \cdot \mathrm{s}^{-1}\right)$ indicates that the UV polymerization process increases the surface charge and hence enhances the fluidic transport. The attractive performance of the new CE microchips has been demonstrated in connection with end-column amperometric and contactless-conductivity detection schemes. While the new approach is demonstrated in connection with PMMA microchips, it could be applied to other materials that undergo light-initiated polymerization. The new approach brings significant simplification of the process of fabricating PMMA devices and should lead to a widespread low-cost production of high-quality separation microchips.

Microfluidic devices are finding numerous applications in analytical chemistry. ${ }^{1,2}$ To address these growing needs, microchip

\footnotetext{
* Corresponding author. E-mail: joewang@nmsu.edu. Tel.: 505-646-2140.

+ New M exico State University.

¥ Research Center J ülich.

$\S$ Academy of Sciences of the Czech Republic.

(1) Becker, H.; Locascio, L. E. Talanta 2002, 56, 267.

(2) Reyes, D. R.; Iossifidis, D.; Auroux, P. A.; M anz, A. Anal. Chem. 2002, 74, 2623.
}

devices have to be mass produced at low cost. Such devices are commonly fabricated with processes originally developed in the microelectronic industry. $3,4 \mathrm{M}$ ost early reports on miniaturized analytical systems have relied on glass or silicon substrates in connection with standard lithographic fabrication technology. However, the cost of producing glass microchip devices has driven researchers and producers to seek alternative materials. Recent efforts have thus led to increasing use of polymeric materials in the preparation of chip-based devices. ${ }^{5}$ Polymeric materials offer attractive mechanical and chemical properties, low cost, ease of fabrication, biocompatibility, and higher flexibility. ${ }^{1}$ Such plastic chips have been fabricated using laser ablation, ${ }^{6}$ plasma etching, ${ }^{7}$ imprinting ${ }^{8}$ hot embossing ${ }^{9}$ injection molding, ${ }^{10}$ and compression molding ${ }^{11}$ techniques. Extensive reviews were published on the analytical applications of polymer microfluidic devices, ${ }^{1}$ and their fabrication techniques. ${ }^{4,5}$ Different polymeric materials, including poly(dimethylsiloxane) (PDMS), ${ }^{12}$ poly(methyl methacrylate) (PM M A),${ }^{9}$ or polycarbonate, ${ }^{11}$ have commonly been employed in the fabrication of microfluidic devices. PM M A has been particularly useful for analytical microsystems, owing to several advantages, including high chemical and mechanical stability and good support of the electroosmotic flow.1,5 Existing methods for fabricating PM M A chips, including direct writing methods $s^{6,7}$ and replication techniques, ${ }^{8-11}$ require specialized and expensive equipment. Replication methods also rely on elevated heat or pressure that can influence the accuracy of the replication. One exception (in term of instrumentation complexity) are wireimprinting methods; ${ }^{8}$ however, such simple low-cost protocols lead to highly irreproducible channels and are limited to very simple

(3) Auroux, P. A.; Iossifidis, D.; Reyes, D. R.; M anz, A. Anal. Chem. 2002, 74 2637.

(4) Becker, H.; Gärtner, C. Electrophoresis 2000, 21, 12.

(5) Soper, S. A.; Ford, S. M.; Qi, S.; M cCarley, R. L.; Kelly, K.; M urphy, M. C. Anal. Chem. 2000, 72, 643A.

(6) Roberts, M. A.; Rossier, J. S.; Bercier, P.; Girault, H. Anal. Chem. 1997 69, 2035.

(7) Rossier, J.; Reymond, F.; M ichel, P. E. Electrophoresis 2002, 23, 858

(8) M artynova, L.; Locascio, L. E.; Gaitan, M .; Kramer, G. W.; Christensen, R. G.; M acCrehan, W. A. Anal. Chem. 1997, 69, 4783.

(9) Ford, S. M.; Kar, B.; McWhorter, S.; Davies, J.; Soper, S. A.; Klopf, M .; Calderon, G.; Saile, V. J. Microcolumn Sep. 1998, 10, 413.

(10) M cCormick, R. M .; Nelson, R. J.; Alonso-Amigo, M. G.; Benvegnu, D. J.; Hooper, H. H. Anal. Chem. 1997, 69, 2626.

(11) Liu, Y.; Ganser, D.; Schneider, A.; Liu, R.; Grodzinski, P.; Kroutchinina, N Anal. Chem. 2001, 73, 4196.

(12) M cD onald, J. C.; Duffy, D. C.; Anderson, J. R.; Chiu, D. T.; Wu, H.; Schueller, O. J. A.; Whitesides, G. M. Electrophoresis 2000, 21, 27.

10.1021/ac035030+ CCC: $\$ 27.50$ (c) 2004 American Chemical Society Published on Web 02/28/2004 


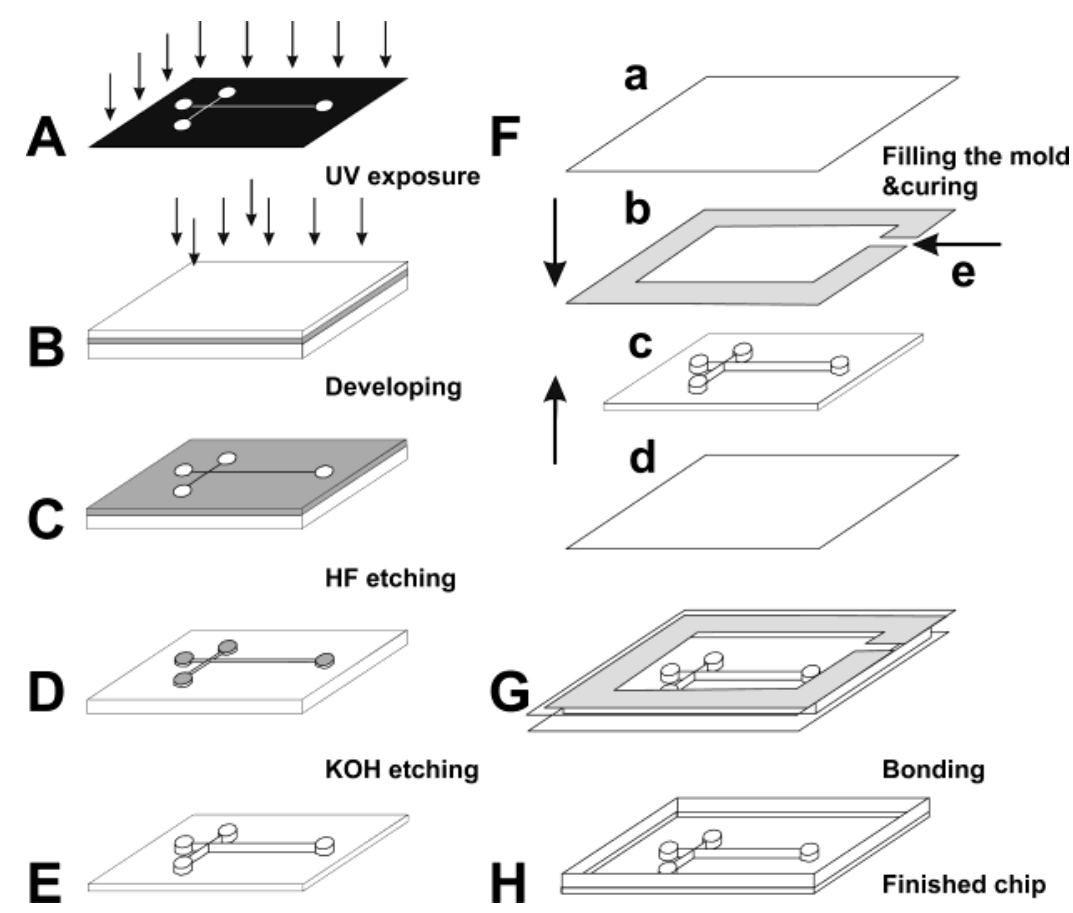

Figure 1. Schematic of the microfabrication process. (A) design of the photolithographic mask; (B) spin-coating the negative photoresist on the oxidized silicon wafer; (C) pattern transfer of the photoresist mask on $\mathrm{SiO}_{2}$ surface; (D) isotropic etching of the $\mathrm{SiO}_{2}$ mask on the $\mathrm{Si}$ surface; (E) anisotropic etching of the Si substrate to raise three-dimensional channel structures; $(F)$ assembling the sandwich mold and molding the monomer solution, (a) glass plate, (b) Teflon spacer, (c) Si master, (d) glass plate, and (e) solution inlet; (G) demolding of the polymerized PMMA substrate with channels; $(\mathrm{H})$ thermal sealing of the two PMMA plates.

geometries. New methods for fabricating polymeric devices are thus needed because existing methods ${ }^{6-11}$ require special replication equipment and are not commonly accessible to most laboratories.

This report describes a simple, user-friendly, and effective method for fabricating high-quality PM M A separation microchips based on an atmospheric pressure molding. The new fabrication route offers a substantial simplification of the entire fabrication process, through a judicious coupling of light-initiated polymerization of methyl methacrylate (M M A) monomer solutions under ambient conditions with common photolithography and wet chemical etching techniques (Figure 1). This approach differs significantly from earlier fabrication schemes of plastic devices in that it does not require elevated pressures and temperatures (and related cooling) or a complicated replication equipment. Since the master is not exposed to excessive pressure and heat (as with imprinting or injection molding), metallization of the silicon masters (aimed at preventing their breakage) is not necessary. UV-initiated polymerization has been shown useful for achieving monolith formation within microchannels, ${ }^{13}$ but not in connection with the preparation of the microchips themselves.

In term of simplicity, the fabrication method described here is similar to the method developed by Whitesides's group for rapid prototyping of PDMS devices ${ }^{12,14}$ (as both do not require special replication equipment and rely on casting the prepolymer solution against a master). However, the new method is not restricted to elastomeric materials and may be readily extended to other

(13) Rohr, T.; Yu, C.; Davey, M. H.; Švec, F.; Fréchet, J. M . J. Electrophoresis 2001, 22, 3959.

(14) Duffy, D. C.; M cD onald J. C.; Schueller O. J. A.; Whitesides, G. M. Anal. Chem. 1998, 70, 4974. polymers that undergo UV-initiated polymerization, thereby extending the scope of polymer microchip fabrication. The ease, simplicity, versatility, and low cost of the new fabrication route thus make it extremely attractive for widespread production of polymeric microchips. The method also enables custom tailoring of the chip composition (and hence the electroosmotic flow, EOF) by the judicious selection of monomers and other reagents. Various aspects of the new method, including the microfabrication efficiency, molding performance of the polymer, master/ template uniformity, microchannel design, and chip-to-chip or run-to-run reproducibility, are reported in the following sections, along with the attractive separation performance of the resulting microchips.

\section{EXPERIMENTAL SECTION}

Microfabrication Procedure. The masters were created by pattern transfer protocol, involving soft photolithography and wet chemical etching. Photolithographic negative masks were designed using a standard computer design software (Adobe Illustrator 10, Adobe) and transferred onto a transparency film at a local photo shop using a commercial high-resolution printer with $3600 \mathrm{dpi}(\sim 7 \mu \mathrm{m})$ resolution (see Figure $1 \mathrm{~A})$. The channel network was represented by $50-\mu \mathrm{m}$-wide transparent lines on a black background. Parallel aligning lines were designed on the negatives for positioning the photomask along the flat of the wafer. Silicon wafers (p-type, $500 \mu \mathrm{m}$ thick, 4-in. diameter, with $\langle 100\rangle$ orientation and 100-nm-thick silicon oxide layer; University Wafers Inc., Weymouth, M A) were used for fabricating the molding tool. A negative photoresist (SU-8 50, M icrochem Corp., N ewton, M A) was spun on the surface of the wafer using a spin-coater (Cee 100, Brewer Science, Rolla, M O) at $3000 \mathrm{rpm}$ for $40 \mathrm{~s}$ with an accelerating ramp of $300 \mathrm{rps}$ (Figure 1B). After a 30-min "prebake" 
in a programmable convection oven (TC-40, SalvisLab), using a thermal gradient from 65 to $95{ }^{\circ} \mathrm{C}\left(5^{\circ} \mathrm{C} / \mathrm{min}\right)$, the photoresist layer was exposed to UV light through the photolithographic mask. The photomask was placed over the spin-coated silicon wafer, and the main separation channel was manually aligned parallel to the primary orientation flat that defines the direction of the etching plane and helps with the orientation during the fabrication process so that only $\langle 111\rangle$ planes are introduced as side walls. The optical mask was secured on the wafer during the exposure by placing on it a $1 / 16$-in.-thick quartz glass plate (essential for the UV exposure), and the system was exposed to UV light for $30 \mathrm{~min}$ ( $365 \mathrm{~nm}, 45 \mathrm{~W} / \mathrm{cm}^{2}$, Phillips). A 40-min "postexposure bake" was carried out using a thermal gradient from 65 to $90^{\circ} \mathrm{C}\left(5^{\circ} \mathrm{C} / \mathrm{min}\right)$. Subsequently, the wafer was developed for $90 \mathrm{~s}$ in a glass Petri dish containing the developer (XP SU-8, $\mathrm{M}$ icrochem Corp.). The unexposed photoresist was cleaned by soaking in acetone and 2-propanol for 30 and 10 s, respectively. Thus, only the desired channel design was photopolymerized on the wafer (Figure 1C). The "hardbake" was carried out by heating the wafers at $150{ }^{\circ} \mathrm{C}$ for $60 \mathrm{~min}$. The wet chemical etching was performed in two steps. First, the exposed silicon oxide layer around the photoresist-covered channel design was isotropically etched in a buffered hydrofluoric acid solution (AF 875-125, Riedelde-Haen, Sigma-Aldrich) for $3.5 \mathrm{~min}$. During this step, only the $\mathrm{SiO}_{2}$ layer was etched to create a $\mathrm{SiO}_{2}$ mask identical to the original photolithographic mask for the second step of etching the silicon substrate (Figure 1D). Subsequently, the wafer was thoroughly rinsed with distilled water and anisotropically etched in a $40 \% \mathrm{KOH}$ solution (containing $5 \% 2$-propanol) at $60{ }^{\circ} \mathrm{C}$ with periodic monitoring until reaching the desired depth (and the corresponding three-dimensional channel structures; Figure $1 \mathrm{E}$ ). The polymerized SU-8 was lifted off during the process of anisotropic etching. This is attributed to the long etching period ( $\sim 40 \mathrm{~min}$ ) at the elevated temperature $\left(60^{\circ} \mathrm{C}\right)$. This did not influence the quality of the anisotropic etching since only the remaining $\mathrm{SiO}_{2}$ surfaces were used as mask.

Mold Design and Molding Procedure. The microfabricated silicon wafer was used as a molding tool representing a structure negative to the desired polymer template. The mold was assembled from a $4 \times 4$ in. glass plate (Figure $1 F, d$ ) to which the molding tool (c) was sealed using a fast-setting epoxy, followed by a square 2-mm-thick Teflon spacer (b) of the same dimensions, with a $16 \times 78 \mathrm{~mm}$ rectangular opening (defining the shape of the plastic chip). A 1-mm inlet was kept open in the spacer to allow the introduction of monomer solutions. The mold was then covered with another glass plate (Figure $1 F$, a) and mechanically sealed using laboratory clamps. The mold, filled with the molding solution (Figure 1F), was exposed to UV light (365-nm lamp, 6 $W$, Cole-Parmer). Complete polymerization of the solution was accomplished within $4 \mathrm{~h}$ under ambient conditions. The silicon ridges of the design were thus transferred to the plastic substrate. Demolding was accomplished by sonicating the mold in water for $10 \mathrm{~min}$ at $40^{\circ} \mathrm{C}$.

The molding solution was prepared by dissolving PM M A beads (200 $\mu \mathrm{m}, \mathrm{FW} 75$ 000, Polysciences Inc., Warrington, PA) in an uninhibited solution of M MA (Polysciences Inc.) at $20 / 80 \mathrm{w} / \mathrm{V}$ ratio at $60{ }^{\circ} \mathrm{C}$. Benzoin methyl ether (BME, $\alpha$-methoxy- $\alpha$ phenylacetophenone, Polysciences Inc.) was added $(0.15 \% \mathrm{w} / \mathrm{V})$ as UV catalyst, promoting the free-radical polymerization of the monomer and the short polymer chains. Prior to pouring the monomer solution into the mold, it was kept in a vacuum for 1 min to avoid bubble formation during the polymerization process.

Thermal Sealing. Prior to sealing, the edges of the structured PM M A channel plate were cut off (with a sharp blade) and $1 / 16^{-}$ in.-diameter access holes were drilled to create reservoir ports. Subsequently, the chips were cleaned by sonication in water and 2-propanol for 2 min and were dried under a stream of nitrogen. The 2-mm-thick PMMA channel plate and the 125- $\mu$ m-thick PMMA cover sheets (GoodFellow, Huntington, U.K.) were sandwiched between two glass plates (of the same dimensions), clamped together using laboratory clamps, and placed in a convection oven for $10 \mathrm{~min}$ at $108^{\circ} \mathrm{C}$. The bonded chip was then allowed to cool slowly and was removed from the clamps (Figure $1 \mathrm{H})$.

Microchip Layout and Characterization.The chip layout had a four-way injection cross that was connected to the three (sample, running buffer, and unused) reservoirs and the separation channel (Figure $1 \mathrm{H}$ ). The microfluidic network consisted of a 54-mm-long separation channel (between the injection cross and the outlet reservoir) and of a 5-mm-long injection channel (between the sample and the injection cross). The channels had a trapezoidal cross section with a top width of $100 \mu \mathrm{m}$, bottom width of $70 \mu \mathrm{m}$, and depth of $20 \mu \mathrm{m}$. Scanning electron micrographs (SEM s) of the silicon masters and of the PMMA templates were obtained with a Hitachi S3200 scanning electron microscope, using an accelerating voltage of $18 \mathrm{kV}$. The PMMA templates were sputtered with a $100-\AA$ thin gold layer and grounded using a thin platinum wire prior to scanning electron microscopy to prevent generation of a static charge on the surface. Gold sputtering of the PM M A chip surfaces was performed with a Denton Vacuum Desk-II, using an argon pressure of 50 mTorr and a current of 40 $\mathrm{mA}$ for $7 \mathrm{~min}$. The quality of the microfabricated chips and the performance of the sealing procedure were monitored using an optical M TV-3 video microscope (Olympus) connected to a CCD/ RGB color video camera (Sony) via a MTV-3 optical interface (Olympus). The profilometer scan of the microchip channel plates was obtained using a Dektak 3030 instrument (Veeco Instruments, Untershliessheim, Germany).

Apparatus. The newly fabricated PM M A chips, as well as the LIGA-based PM MA and glass chips [used for comparison, from the Institut für M ikrotechnik ( $M$ ainz, Germany) ${ }^{15}$ and $M$ icralyne (model MC-BF4-001, Edmonton, Canada), respectively], had simple cross layouts, with the four-way injection cross connected to the sample, running buffer, and unused reservoirs and the separation channel, in a manner similar to the atmospheric-molded PM M A chip (Figure 1H). The commercial PM M A chip consisted of a 48-mm-long separation channel (between the injection cross and the waste reservoir) and a 9-mm-long injection channel (from the sample reservoir and the injection cross). The channels had a $50 \times 50 \mu \mathrm{m}$ squared cross section. The glass microchip had a 54 -mm-long separation channel, with a $50 \times 20 \mu \mathrm{m}$ ellipsoidal cross section. For conductivity detection, the electrodes were placed on the separation channel (in a contactless mode) $3 \mathrm{~mm}$ away

(15) Wang, J.; Pumera, M.; Chatrathi, M. P.; Escarpa, A.; Konrad, R.; Griebel, A.; Dörner, W.; Löwe, H. Electrophoresis 2002, 23, 596. 
from the waste reservoir. ${ }^{16}$ For amperometric detection, the waste reservoir was cut off and the chips were placed in a laboratorybuilt Plexiglas holder in which the detection and high-voltage electrodes were configured. Details of the system integration with the amperometric detection were published elsewhere. ${ }^{17}$ The homemade high-voltage power supply had an adjustable voltage range between 0 and $+4000 \mathrm{~V}$; platinum wires (placed in the holder reservoirs) established the contact to the power supply. Short pipet tips were inserted to the reservoirs of the chip to establish solution contact between the channels on the chip and corresponding reservoirs on the chip holder.

Amperometric detection was performed with an electrochemical analyzer 1232 ( $\mathrm{CH}$ Instruments Inc., Austin, TX) using the "amperometric i-t curve" mode. Thick-film screen-printed carbon working, platinum auxiliary, and $\mathrm{Ag} / \mathrm{AgCl}$ reference wire electrodes were used for the end-column detection. ${ }^{17}$

A capacitively coupled contactless conductivity detector (CCD) was used for the detection of ionic species. ${ }^{16,18}$ The circuit contained a RC filter (time constant, $0.01 \mathrm{~s}$ ), followed by a voltage follower (LF 356) to the circuit output, and allowed convenient data reading. A HP 8116A function generator (Hewlett-Packard, Palo Alto, CA) was used for generating the sinusoidal signal. The electronic circuit was placed in a shielding box to protect the electronics from external electric fields. The open side of the box was placed as close as possible to the detection reservoir, to act also as a shield for the sensing electrode system. Details of the fabrication of the CCD electrode were reported earlier. ${ }^{18}$ B riefly, rectangular-shaped electrodes $(0.8 \mathrm{~mm} \times 24 \mathrm{~mm})$ were made from two $10-\mu$ m-thick aluminum foil strips, placed in an "antiparallel" orientation. Thin copper wires were attached to the electrodes on the top of the detector plate using a conducting epoxy (Chemtronics, Kennesaw, GA) and were tin-soldered to the detector electronics. A PCI-6035E 200 kS/ s, 16 bit A/ D board with NI-DAQ driver software for WIN 2000/ NT and a program written in LabView 6.0.2 software (all National Instruments, Austin, TX) were used for the data collection. The electronic components were purchased from local suppliers.

Reagents. Dopamine, catechol, histidine (His), 2-(N-morpholino)ethanesulfonic acid (MES), methylamine, ammonium chloride, sodium chloride, ammonium nitrate, and sodium perchlorate were purchased from Sigma. The analysis of neurotransmitters was performed with a $20 \mathrm{mM}$ M ES buffer ( $\mathrm{pH}$ 6.5). The run buffer for separation of low-ionic explosives was a MES/ His buffer (20 $\mathrm{mM}$ each, $\mathrm{pH}$ 6.1). Stock solutions of neurotransmitters (dopamine and catechol, $10 \mathrm{mM}$ ) and of the target ions (ammonium, methylammonium, and sodium chloride, nitrate, and perchlorate, $100 \mathrm{mM}$ ) were prepared daily by dissolving the analytes or corresponding salts in the run buffer. All chemicals were used without any further purification.

Electrophoretic Procedure. The channels of the new PM M A chip, as well as those of the commercial chips (used for comparison), were treated before use by rinsing with $0.1 \mathrm{M}$ sodium hydroxide and deionized water for $10 \mathrm{~min}$ each. The sample mixture was added to the sample reservoir, while the other

(16) Pumera, M .; Wang, J.; Opekar, F.; Jelínek, I.; Feldman, J.; Löwe, H.; Hardt, S. Anal. Chem. 2002, 74, 1968.

(17) Wang, J.; Tian, B.; Sahlin, E. Anal. Chem, 1999, 71, 5436.

(18) Tůma, P.; Opekar, F.; J elínek, I. Electroanalysis 2001, 12, 989. reservoirs were filled with the electrophoretic run buffer. Sample injections were performed after stabilization of the detector baseline by applying a potential of $1500 \mathrm{~V}$ for $3 \mathrm{~s}$ and $500 \mathrm{~V}$ for is (for amperometric and conductivity experiments, respectively) between the sample reservoir and the detection (ground) reservoir. This drove the sample zone into the separation channel through the intersection. The analytical separation proceeded by applying the separation voltage to the run buffer reservoir with the detection reservoir grounded and all other reservoirs floating.

Safety Considerations. Methyl methacrylate and benzoin methyl ether are toxic substances and other used chemicals are irritants. Accidental inhalation, ingestion, or skin and eye contact with these chemicals should be avoided. Hydrofluoric acid and concentrated potassium hydroxide solutions are highly corrosive. The etching of silicon substrates should be carried out in a ventilated fume hood using proper double-layered nitrile gloves, apron, goggles and a protective shield. The high-voltage power supply can cause an electrical shock and hence should be handled with extreme care.

\section{RESULTS AND DISCUSSION}

The new fabrication route offers a substantial simplification of the fabrication of PM M A microchips, through the coupling of lightinitiated polymerization under ambient conditions with common pattern transfer (photolithography and wet chemical etching) techniques (Figure 1).The new simplified fabrication route provides high-quality polymeric devices with excellent accuracy of replication of the silicon structures to PM M A. Scanning electron microscopy views of a typical four-way injection-cross section of the positive-relief silicon master $(A)$ and of the resulting PM M A chip (B) are shown in Figure 2. The pattern transfer of the mask design onto the silicon wafer results in a well-defined, sharp and symmetric geometry. Such high-quality master structure indicates that the printer resolution ( $3600 \mathrm{dpi}$ ) is satisfactory for generating the channel and intersection patterns on the photolithographic mask (as a consequence of having an orthogonal geometry resulting in straight rows and column pixels with the printer). The raised "positive ridges" of channels are formed by the top planar $\langle 100\rangle$ orientation layers and the side $\langle 111\rangle$ orientation silicon walls. The replica of these silicon structures in PM MA is shown in Figure 2B. The trapezoidal shape negative relief channel structures are well defined, and the molded surfaces are highly smoothed with minimal roughness (reflecting the quality of the silicon master and accuracy of the replication process). In contrast to imprinting techniques where the plastic substrates are heated to temperatures above $T_{g}$, the free flow of the "prepolymer" solution takes shape around the solid structure as it is being cured from a solution into a hard polymer. Such procedure results in a structurally stress-free work piece following the demolding, in a manner similar to compression or injection molding techniques; ${ }^{10,11}$ however, this is accomplished without specialized molding-replication equipment, heating or electroforming of metal masters.

The structure transfer into the PM M A material is characterized by high precision of the master replication. Nearly 100 identical separation chips have thus been produced from a single master. The reproducibility of such replica molding was studied for eight separate chips, templated from the same molding silicon master, and selected randomly from a pool of 25 different chips. Table 1 

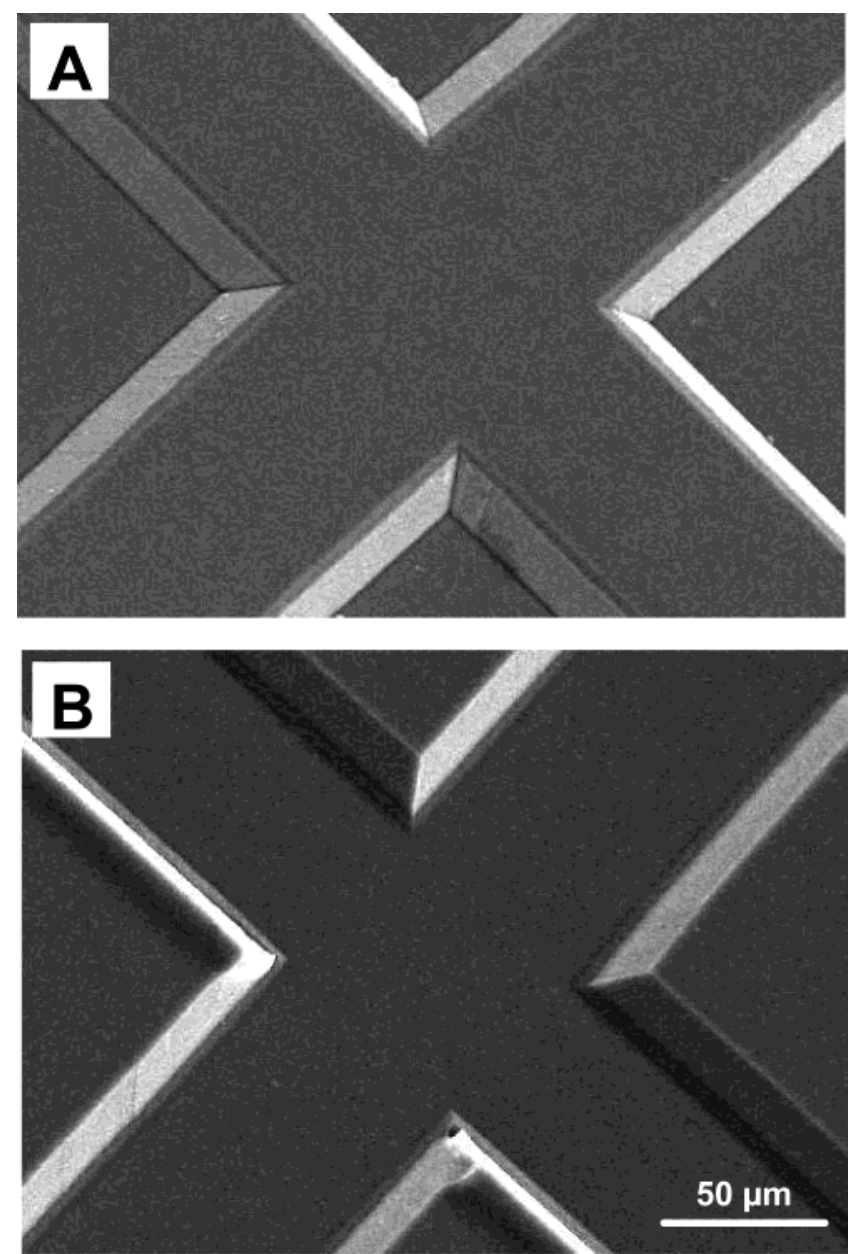

Figure 2. Scanning electron micrographs of the injection-cross section of the silicon molding master $(\mathrm{A})$ and of the resulting PMMA channel template (B) after atmospheric pressure molding. Conditions: accelerating voltage, $18 \mathrm{kV}$; magnification, $\times 400$.

\section{Table 1. Replication of Microchannels by Atmospheric Pressure Molding: RSDs of Eight Microchips Replicated from a Single Molding Tool ${ }^{a}$}

microchip no.

$$
\begin{aligned}
& 1 \\
& 2 \\
& 3 \\
& 4 \\
& 5 \\
& 6 \\
& 7 \\
& 8
\end{aligned}
$$$$
\text { RSD (\%) }
$$

$\mathrm{w}_{\mathrm{a}}(\mu \mathrm{m})$
69
69
69
71
69
71
69
70

1.32

$\mathrm{w}_{\mathrm{b}}(\mu \mathrm{m})$
97
95
96
99
97
96
97
99

1.46
$\mathrm{D}(\mu \mathrm{m})$

20

18

19

20

20

18

20

20

a Channel bottom width $\left(w_{a}\right)$, channel top width $\left(w_{b}\right)$, and calculated depth (D) measurements taken on the separation channel $50 \mu \mathrm{m}$ from the injection cross (after sealing the chips).

summarizes the chip-to-chip reproducibility of the finished chips. Low relative standard deviation (RSD) values of 1.32, 1.46, and $4.73 \%$ were observed for the channel bottom width, the top width, and the depth, respectively. The exact reason for the larger RSD for the channel depth is not fully understood.

Due to the trapezoidal shape of the channels, the molding tools are easily demoldable by simple sonication. It should be mentioned

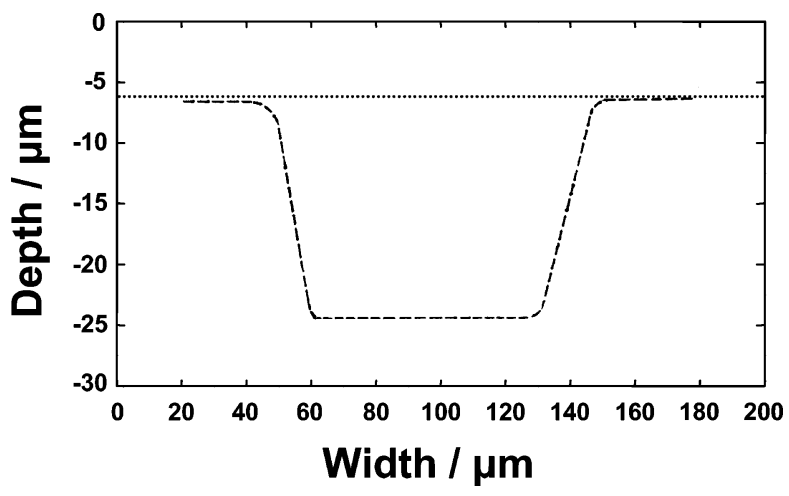

Figure 3. Corrected profilometer scan across the channel plate of the atmospheric pressure-molded PMMA microchip, recorded at the injection cross after demolding from the silicon master.

that, in contrast to the imprinting methods, ${ }^{8}$ no change in the geometry occurs after demolding of the chips. Furthermore, no breaking of the molding tools takes place as no pressure or elevated temperature is applied during the molding. Thus, relaxation of the polymeric substrates (associated with the removal of weight, pressure, or heat) and related changes in the channel geometry and dimensions are avoided.

The plastic structure is characterized by trapezoidal channels with well-defined walls. The top and bottom widths and the depth of the plastic structure (101, 71 , and $18 \mu \mathrm{m}$, respectively) are nearly identical with the corresponding dimensions of the silicon master (97, 70 , and $19 \mu \mathrm{m}$, respectively), indicating the high replication accuracy. The discrepancy (of 9-10 $\mu \mathrm{m}$ ) reflects the finite dimensions of the stylus tip (of $5-\mu \mathrm{m}$ radius) touching the left and right sides of the microchannel during the scan. This discrepancy is adjusted and shown in Figure 3 as a corrected profilometer scan of the PM MA channel plate after demolding from the silicon master. Overall, the nearly identical dimensions of the silicon master and the PMMA channel indicate good reproduction, with high replication accuracy. Such data compare favorably to those of other molding and imprinting techniques. 8,10 The slight asymmetry in the side walls of the PM MA channel is also an artifact of the profilometer instrument, ${ }^{10}$ as confirmed by the highly symmetrical trapezoidal features of the SEM images (Figure 2).

Initial atmospheric molding experiments were performed using different mixtures of uninhibited MMA solutions with the BME polymerization catalyst. The amount of the UV catalyst was varied between 0.05 and $0.5 \% \mathrm{w} / \mathrm{v}$ range. A $0.15 \% \mathrm{BME}$ level proved to be optimal for complete curing of the substrates within less than $4 \mathrm{~h}$, while providing high-quality structures. Higher BME levels resulted in faster curing but led to uncontrolled polymerization and related excessive heating. To prevent volume shrinkage during molding, it is essential to increase the density of the molding solution. This can be accomplished by either exposing the monomer to UV light (for $\sim 1 \mathrm{~h}$ with proper stirring) or preparing a prepolymer solution by dissolving PM M A beads in the uninhibited monomer solution (at $20 \% \mathrm{w} / \mathrm{v}$ ).

Thermal bonding was used as it leads to homogeneous surface charge. ${ }^{19}$ Other bonding techniques, such as solvent bonding or lamination (with pressure-sensitive PM M A tapes), are less satis-

(19) Woolley, A. T.; Kelly, R. T. Anal. Chem. 2003, 75, 1941. 


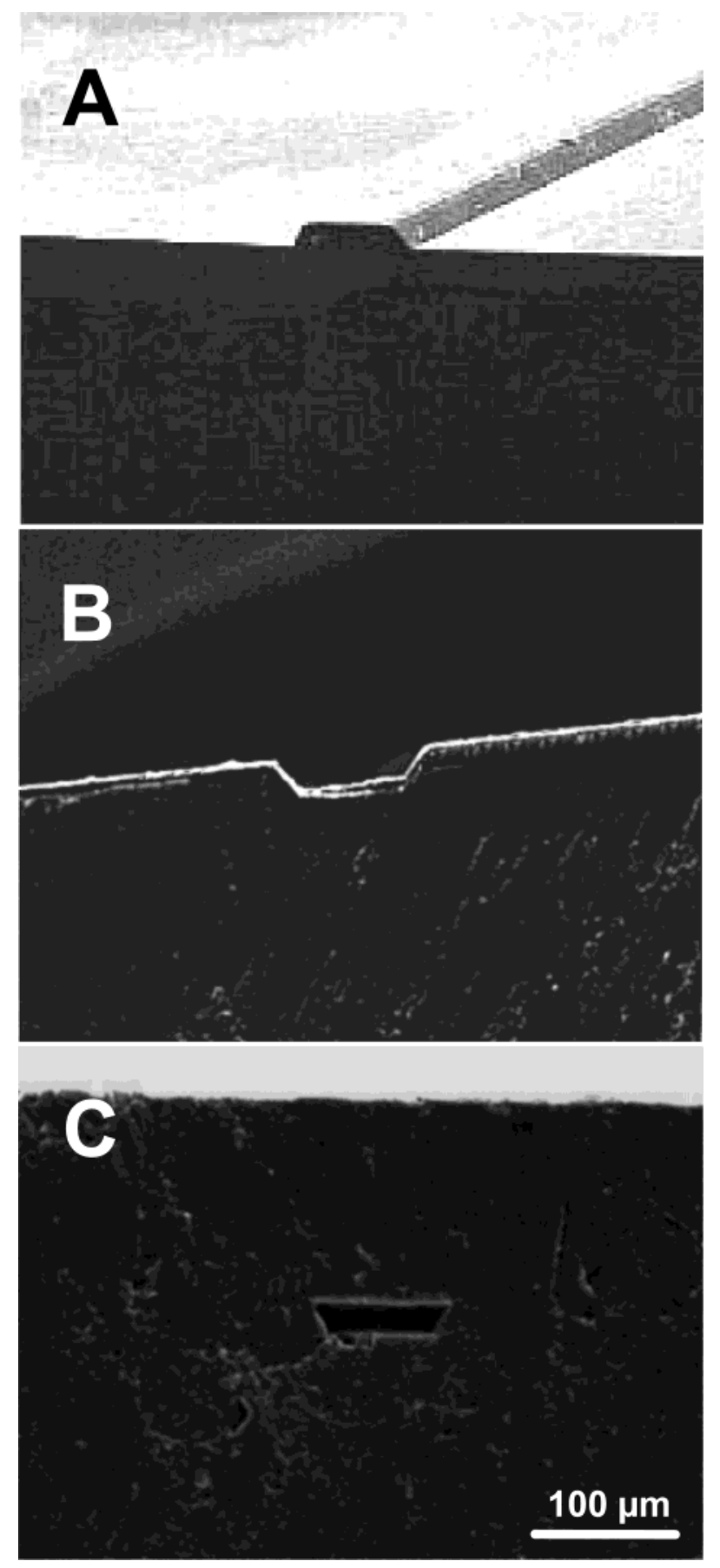

Figure 4. Scanning electron micrographs of $(A)$ the outlet section of the channel ridge structure of the silicon master, $(B)$ the corresponding template in PMMA substrate, and $(C)$ the complete microchip channel outlet after thermal bonding $\left(10 \mathrm{~min}\right.$ at $108{ }^{\circ} \mathrm{C}$. Conditions: accelerating voltage, $18 \mathrm{kV}$; magnification, $\times 250$.

factory, due to possible health risks of the 2,4-pentadione solvent or swelling of the pressure-sensitive adhesive of the laminated chips, respectively. Thermal bonding of the channel and cover plates (clamped between two glass plates and placed horizontally) in a conventional oven at $108{ }^{\circ} \mathrm{C}$ for 10 min resulted in good sealing without any deleterious effects. Figure $4 \mathrm{~A}$ displays SEM images of a raised silicon trapezoidal microstructure of the channel outlet, while Figure $4 \mathrm{~B}$ displays the corresponding channel template on the PM M A substrate. Such images indicate again that the replica molding results in high-quality structures without any change in the channel geometry. The resulting channel structure in the PM M A channel plate (Figure 4B) exhibits highly smoothed wall surfaces. The channel outlet, after thermal bonding, is displayed in Figure 4C. For optimal bonding, the thicker channel plate was placed on top of the cover plate (both being sandwiched between two glass plates of the same dimensions). Such a process provided no observable boundaries between the two plates along with the absence of voids (associated with the air bubbles entrapped during the bonding process). In contrast, when the cover plate was placed on top of the channel plate, thermal collapsing of the cover plate was observed, possibly due to the thermal relaxation of the thin $(\sim 125 \mu \mathrm{m})$ commercial PMMA sheets at this temperature.

The EOF properties of the newly fabricated device were evaluated for untreated surfaces, using the well-established current monitoring technique of Huang et al. ${ }^{20}$ The EOF was nearly independent of the solution $\mathrm{pH}$, changing only slightly from $2.0 \times 10^{-4}$ to $2.2 \times 10^{-4} \mathrm{~cm}^{2} \cdot \mathrm{V}^{-1} \cdot \mathrm{s}^{-1}$ over the $3.5-9.5 \mathrm{pH}$ range (indicating a wide operational $\mathrm{pH}$ range). The EOF value calculated at pH 6.5 (with a field strength of $270 \mathrm{~V} \cdot \mathrm{cm}^{-1}$ ), $2.12 \times 10^{-4}$ $\mathrm{cm}^{2} \cdot \mathrm{V}^{-1} \cdot \mathrm{s}^{-1}$, compares favorably with the EOF value of the LIGAbased PM M A chip $\left(1.82 \times 10^{-4} \mathrm{~cm}^{2} \cdot \mathrm{V}^{-1} \cdot \mathrm{s}^{-1}\right)$ and with literature values for bare PM M A chips $\left((1.2-2.6) \times 10^{-4} \mathrm{~cm}^{2} \cdot \mathrm{V}^{-1} \cdot \mathrm{s}^{-1}\right),{ }^{5,21}$ The slightly higher EOF value observed with the atmosphericmolded chips indicates that the UV polymerization process increases the surface charge and hence enhances the fluidic transport of the microchips (in comparison to other fabrication processes). The modest increase in the EOF value is not uncommon, as similar observations were reported earlier in connection with UV laser subablation. ${ }^{22}$ The higher charge density is attributed to partial oxidation of carbon bonds on the surface of the exposed polymer during the molding. Simple activation with $0.1 \mathrm{M} \mathrm{NaOH}$ can further increase the surface charge and enhance the electroosmotic pumping. An EOF value of $2.71 \times 10^{-4}$ $\mathrm{Cm}^{2} \cdot \mathrm{V}^{-1} \cdot \mathrm{s}^{-1}$ was estimated after a 10-min alkaline treatment (with $0.1 \mathrm{M} \mathrm{NaOH}$ ), followed by $10 \mathrm{~min}$ of flushing with deionized water. The additional activation reflects the partial hydrolysis of the methyl ester bonds of the polymer chain.

The analytical performance of the atmospheric-molded PM M A microchips was demonstrated in connection with the separation of organic molecules and ionic species coupled to end-column amperometric and contactless-conductivity detection schemes. The separation performance of both detection schemes is summarized in Table 2. Catecholamine analytes are commonly used for assessing the separation in microfluidic devices in connection with amperometric detection. $16,23,24$ As indicated from Figure $5 A$, the PM M A microchip provides baseline-resolved well-defined peaks for $100 \mu \mathrm{M}$ dopamine and catechol within less than $80 \mathrm{~s}$. Some tailing is observed for the positively charged dopamine. The migration times of both analytes in the PMMA channel are

(20) Huang, X.; Gordon, M .; Zare, R. N. Anal. Chem. 1988, 60, 1837.

(21) Soper, S. A.; Henry, A. C.; Vaidya, B.; Galloway, M .; Wabuyele, M .; M cCarley, R. L. Anal. Chim. Acta 2002, 470, 87.

(22) Johnson, T. J.; Ross, D.; Gaintan, M.; Locascio, L. E. Anal. Chem. 2001, $73,3656$.

(23) Baldwin, R. P.; Roussel, T. J.; Crain, M . M .; Bathlagunda, V.; J ackson, D. J.; Gullapalli, J.; Conklin, J. A.; Pai, R.; Naber, J. F.; Walsh, K. M .; Keynton, R. S. Anal. Chem, 2002, 74, 3690.

(24) Chen, D. C.; Hsu, F. L.; Zhan, D. Z.; Chen, Ch. H. Anal. Chem, 2001, 73, 758. 
Table 2. Comparison of the Separation Performance of the Atmospheric Molded PMMA Microchip with Those of Commercial PMMA and Glass Chipsa

\begin{tabular}{llll} 
parameter & $\begin{array}{c}\text { molded } \\
\text { PM M A chip }\end{array}$ & \multicolumn{1}{c}{$\begin{array}{c}\text { glass } \\
\text { chip }\end{array}$} & $\begin{array}{l}\text { commercial } \\
\text { PM M A chip }\end{array}$ \\
$t_{\mathrm{DA}}$ & $34.7 \mathrm{~s}$ & $31.9 \mathrm{~s}$ & $43.8 \mathrm{~s}$ \\
$\mathrm{t}_{\mathrm{CA}}$ & $79.8 \mathrm{~s}$ & $51.5 \mathrm{~s}$ & $136.1 \mathrm{~s}$ \\
$\mathrm{t}_{\mathrm{NH}_{4}}$ & $27.7 \mathrm{~s}$ & & $34.9 \mathrm{~s}$ \\
$\mathrm{t}_{\mathrm{M} \mathrm{NH}}$ & $32.8 \mathrm{~S}$ & & $43.1 \mathrm{~s}$ \\
$\mathrm{t}_{\mathrm{Na}}$ & $37.5 \mathrm{~S}$ & & $49.25 \mathrm{~s}$ \\
$\mathrm{~N}_{\mathrm{DA}}$ & $4402 \mathrm{~m}^{-1}$ & $19752 \mathrm{~m}^{-1}$ & $4073 \mathrm{~m}^{-1}$ \\
$\mathrm{~N}_{\mathrm{CA}}$ & $26156 \mathrm{~m}^{-1}$ & $8885 \mathrm{~m}^{-1}$ & $9996 \mathrm{~m}^{-1}$ \\
$\mathrm{~N}_{\mathrm{NH}}$ & $20703 \mathrm{~m}^{-1}$ & & $42775 \mathrm{~m}^{-1}$ \\
$\mathrm{~N}_{\mathrm{MeNH}}$ & $34481 \mathrm{~m}^{-1}$ & & $49918 \mathrm{~m}^{-1}$ \\
$\mathrm{~N}_{\mathrm{Na}}$ & $34704 \mathrm{~m}^{-1}$ & & $41551 \mathrm{~m}^{-1}$ \\
$\mathrm{R}_{\mathrm{DA}-\mathrm{CA}}$ & 2.04 & 1.51 & 2.74 \\
$\mathrm{R}_{\mathrm{M} \mathrm{eNH}}-\mathrm{Na}$ & 1.12 & & 1.20
\end{tabular}

a Separation of $100 \mu \mathrm{M}$ dopamine (DA) and catechol (CA) performed using the conditions of Figure 5; separation of $2 \mathrm{mM}$ ammonium, $1 \mathrm{mM}$ methyl ammonium $\left(\mathrm{MeNH}_{3}\right)$, and $1 \mathrm{mM}$ sodium, performed using the conditions of Figure 6.

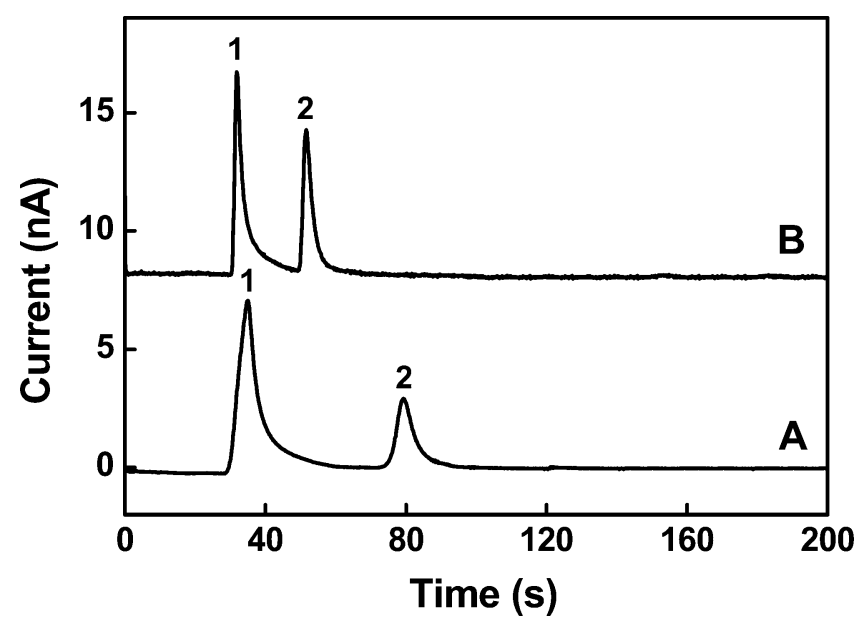

Figure 5. Electropherograms obtained at the atmospheric pressuremolded (A) and commercial glass (B) chips for a mixture of $100 \mu \mathrm{M}$ dopamine (1) and catechol (2). Amperometric detection using a potential of $+0.70 \mathrm{~V}$ (vs Ag/AgCl wire). Run buffer, MES, $20 \mathrm{mM}, \mathrm{pH}$ 6.5; separation and injection voltages, $+1500 \mathrm{~V}$; injection time, $2(\mathrm{~A})$ and $3 \mathrm{~s}(\mathrm{~B})$.

relatively longer than those observed with the commercial glass chips (Figure 5B). However, both PM M A and glass chips exhibit a similar separation performance. The resolution of the two compounds was higher on the PM M A chip (2.04 vs 1.52). The number of theoretical plates for dopamine was determined to be greater than 4400 plates/ $\mathrm{m}$; such value compares favorably with that of the LIGA-based PM M A chip ( 4100 plates $/ \mathrm{m}$ ) and is $\sim 5$ times lower than the number estimated for the glass microchannel. Note that the amperometric detector of both (glass and PM M A) chips was placed outside the separation channel, leading to additional band broadening and hence to lower plate numbers. The number of theoretical plates for neutral catechol was found to be approximately 3 - and 2.5 -fold higher than those observed at the commercial glass and plastic chips, respectively. The number of theoretical plates for the cationic dopamine is slightly higher than that of the commercial PMMA chip (4402 vs $4073 \mathrm{~m}^{-1}$, respectively) but is significantly lower than that $\left(19752 \mathrm{~m}^{-1}\right)$ observed with the glass chip. This difference appears to reflect

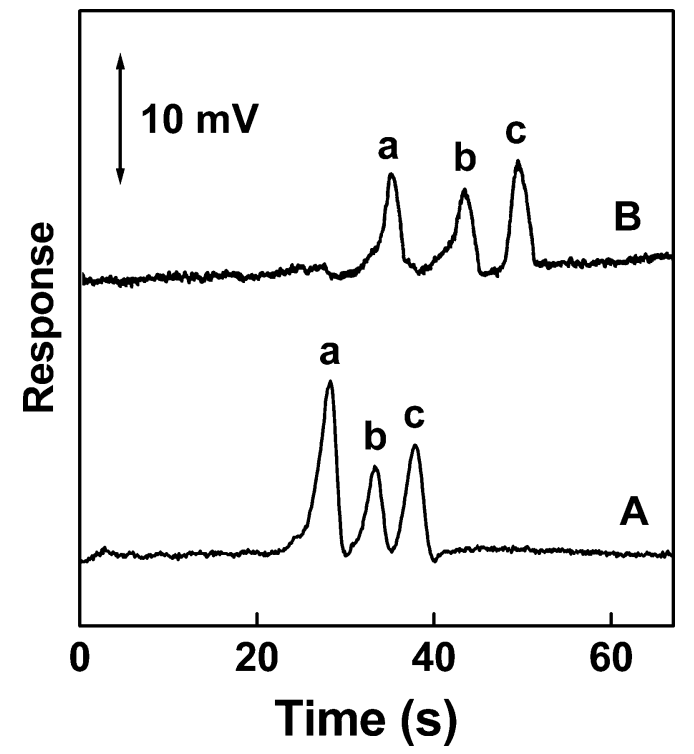

Figure 6. Electropherograms for a mixture solution containing 2 $\mathrm{mM}$ ammonium (a), $1 \mathrm{mM}$ methylammonium (b), and $1 \mathrm{mM}$ sodium (c) cations obtained with the atmospheric pressure-molded $(A)$ and the LIGA-based (B) PMMA chips. Operation conditions: separation voltage, $+1000 \mathrm{~V}$; injection voltage, $+500 \mathrm{~V}$; injection time, $1 \mathrm{~s}$; run buffer, $20 \mathrm{mM} \mathrm{MES/His} \mathrm{(} \mathrm{pH}$ 6.1); contactless conductivity detection with a frequency of $200 \mathrm{kHz}$ and a peak-to-peak voltage of $10 \mathrm{~V}$.

the interaction of the charged neurotransmitter with the channel wall that results in higher peak tailing. The plate numbers obtained for ammonium, methylammonium, and sodium were higher using the commercial chips compared to the molded one. The different channel geometries and dimensions of the two PM M A chips may account for the different plate numbers. Figure 6 demonstrates the performance of the new PM M A chips in connection with the separation and conductivity detection of explosive-related ions. It compares the conductivity CE response of a mixture containing $2 \mathrm{mM}$ ammonium, $1 \mathrm{mM}$ methylammonium, and $1 \mathrm{mM}$ sodium cations at the atmospheric-pressure molded (A) and the LIGAbased commercial (B) PM M A chips. These cations are baseline separated within less than 1 min. The molded PMMA chips yielded higher fluidic transport and shorter analysis times than the commercial PMMA chip. The newly fabricated chips also provided an improved peak shape and high plate numbers ( 20700 , 34 500, and 34700 plates/ $\mathrm{m}$ for ammonium, methylammonium, and sodium, respectively).

\section{CONCLUSIONS}

This report has demonstrated a new low-cost, user-friendly approach for rapid prototyping of polymeric CE chip devices, based on atmospheric pressure molding. The new method brings significant simplification of the process of fabricating PMMA devices, without compromising the quality and accuracy of the channel replication. This approach greatly reduces the complexity and cost of prototyping and manufacturing, as it does not require complicated replication equipment, metallization of the molding tools, or elevated temperatures and pressures. The new approach is applicable to a wide range of elastomeric and nonelastomeric materials that undergo UV-initiated polymerization. Due to its simplicity, low cost, and wide scope, the new fabrication route is expected to facilitate the fabrication of separation microsystems 
and comparison of prototypes in different laboratories. While the new fabrication method has been demonstrated in connection with separation chips, it can be readily extended to other planar PM M A structures.

A major and distinct advantage of the new method is that the surface chemistries can be modified during the fabrication process itself. Such tailoring cannot be accomplished by common "embossing" fabrication strategies that rely on cast sheets of polymers. Ongoing experiments in our laboratory thus focus on tailoring (enhancing and reversing) the electroosmotic flow properties of the new PMMA chips (via a judicious choice of the monomer material). For example, we observed a nearly 2-fold increase of the EOF and a reversal of the EOF by adding methylacrylic acid and aminoethyl moieties, respectively, to the monomer solution. These results and other aspects of the bulk modification route will be reported separately.

The new procedure is somewhat limited in its ability to fabricate high aspect ratio structures. While we are able to fabricate channels widths down to $35 \mu \mathrm{m}$, producing higher aspect ratios would require further optimization of the viscosity of the polymerization solution and possible use of a vacuum chamber. These issues are currently being examined, as well as the fabrication of more complex chip geometries containing additional functional elements.

\section{ACKNOWLEDGMENT}

This research was supported by grants from Department of Homeland Defense (M IPT Project 2002-J-A-139) and EPA (Award RD-8309001-0). We gratefully acknowledge A. J. Ibáñez for valuable discussions.

Received for review September 1, 2003. Accepted January 26, 2003.

$\mathrm{AC035030+}$ 